\title{
Fabrication of a high hydrophobic PVDF membrane via nonsolvent induced phase separation
}

\author{
Chun-Yin Kuo ${ }^{\mathrm{a}}$, Hui-Ni Lin ${ }^{\mathrm{a}}$, Hui-An Tsai ${ }^{\mathrm{a}, \mathrm{b} *}$, \\ Da-Ming Wang ${ }^{\mathrm{a}, \mathrm{c}}$, Juin-Yih Lai ${ }^{\mathrm{a}}$ \\ ${ }^{a}$ R\&D Center of Membrane Technology, Chung Yuan University, Chung-Li 320, Taiwan \\ ${ }^{b}$ Department of Material and Fiber, Nanya Institute of Technology, Chung-Li 320, Taiwan \\ Tel.+886-3-4361070, ext.4101; Fax+886-3-4651975; email: huian@nanya.edu.tw \\ ${ }^{c}$ Department of Chemical Engineering, National Taiwan University, Taipei, Taiwan
}

Received 26 July 2007; accepted revised 21 September 2007

\begin{abstract}
The aim of this paper is to prepare a PVDF (polyvinylidene fluoride) microporous membrane by using alcohol as nonsolvent (coagulant). A porous membrane with high hydrophobic surface (water contact angle $\sim 148^{\circ}$ ) was facilely created by wet immersion method without modification with special low surface energy compounds and the need of special instruments. In addition, the PVDF casting film was passed through alcohol bath for $2 \mathrm{~s}$ and then immersed into water precipitation bath, the porous PVDF membrane with hydrophobic surface (water contact angle $\sim 135^{\circ}$ ) was also formed. The characterization of the porous PVDF membrane obtained from various coagulation conditions was carried out by a pore size analysis and a direct contact membrane distillation (DCMD). The permeation flux and rejection coefficient of the porous PVDF membrane fabricated via dual-bath coagulated was close to commercial one.
\end{abstract}

Keywords: Hydrophobic; PVDF; DCMD; Phase inversion; Microporous

\section{Introduction}

In membrane separation fields, hydrophobic porous membranes are widely applied in membrane distillation (MD), supported liquid membrane (SLM), osmotic distillation (OD)

*Corresponding author. and membrane contact (MC) [1]. Membrane distillation (MD) is generally used to remove water from aqueous solutions of inorganic solutes. In this field, desalination is the best-known for MD application. Although the concept of MD has been known for about 40 years, the process is still being developed. Among the four membrane

Presented at the Fourth Conference of Aseanian Membrane Society (AMS 4), 16-18 August 2007, Taipei, Taiwan. 
distillation (MD) configuration was shown below: (A) direct contact membrane distillation (DCMD), (B) air gap membrane distillation (AGMD), (C) sweeping gas membrane distillation (SGMD), (D) vacuum membrane distillation (VMD). DCMD is the most widely used.

Direct contact membrane distillation is one of the emerging non-isothermal membrane separation processes. This process refers to a thermally driven transport of water through microporous hydrophobic membrane. The membrane is maintained between a feed side and a permeate side. The hydrophobic property of the porous membrane can prevent the aqueous solution penetration into the membrane pores. Thus, the higher hydrophobicity and larger pores of the membrane with high permeate flux under a high rejection will be expected.

The hydrophobicity of a surface can be enhanced by chemical modification that can lower the surface energy or by the introduction of proper surface roughness. The mainly techniques to fabricate the hydrophobic porous membrane as follows: stretching [2], thermally induced phase separation (TIPS) [3-6] and nonsolvent induced phase separation (NIPS) [7-11]. Commercially available PTFE and PP membranes usually produced by stretching or thermal method, but the preparation procedure is complicated and high cost. Compared with other method, the NIPS is a simple and convenient method to prepare porous membrane.

PVDF is a commercially available fluoropolymer with low surface energy and good physical, chemical, and mechanical properties. Therefore, it is reasonable to expect that the PVDF membrane with a porous surface structure should have enhanced hydrophobicity. As far as we know, few study paid attention on the surface properties of the membrane. A symmetric porous PVDF membrane can be obtained by using a modified phase inversion method using a soft precipitation bath of water/DMAc mixture with the ratio smaller than $50 / 50$, and by using a gelation-in-the-air method instead of the immersion-precipitation phase inversion method. The top surface of the sponge-like porous PVDF membrane exhibited greatly enhanced hydrophobicity, having a water contact angle (CA) from 140 to $151^{\circ}$ [12]. However, the separation efficiency of the high hydrophobic PVDF membrane was not discussion. In this paper, a high hydrophobic PVDF membrane was fabricated by using alcohol as coagulant via wet immersion method. A high hydrophobic surface $\left(\sim 140^{\circ}\right)$ and porosity of PVDF membrane was formed by passing through the 1 st coagulant of alcohol for $2 \mathrm{~s}$ and then immersed into water precipitation bath. The hydrophobic PVDF membrane formed without modification with special low surface energy compounds and the need of special instruments. The porous PVDF membranes were also carried out by a direct contact membrane distillation (DCMD). The permeate flux and rejection coefficient of the porous PVDF membranes were investigated in this study.

\section{Experimental}

\subsection{Materials}

The polymer used in this study was PVDF (Polyvinylidene fluoride) purchased from Elf Atochem North America, Inc. (Kynar 760). Anhydrous N-methyl-2-pyrrolidinone (NMP) supplied from Aldrich was used as the solvent without any further purification. The dope solution was prepared at room temperature by dissolving polymer in NMP for 3 days. The obtained solution was kept at room temperature for $24 \mathrm{~h}$ to degass.

\subsection{Membrane preparation}

The polymer solution was cast on a glass plate with the thickness of $300 \mu \mathrm{m}$ by a Gardner knife. The nascent membrane was immersed into alcohol coagulants (methanol, ethanol, $n$-propanol and $n$-butanol) for $2 \mathrm{~s}$, and then was immersed 
in water coagulation bath (called dual coagulation method) or only immersed into alcohol bath for 1 day to precipitate the polymer. After precipitation, the membranes were peeled off and further dried in air for 1-2 days.

\subsection{Water contact angle measurement}

Water contact angle of membrane surface was measured by using sessile drop method and all the contact angle data were an average of ten measurements on different locations of membrane surface. A water droplet was introduced on the surface of membranes, and the contour of the water drop was recorded by using an automatic interfacial tensiometer (Kyowa Interface Science, PD-VP).

\subsection{Light transmission}

Light transmission experiments were performed to measure the time of the onset of liquid-liquid demixing in the casting solution. The principle of light transmission experiments is that the light transmittance of the casting solution would decrease with the appearance of optical inhomogenities, which can be induced by liquidliquid demixing. Therefore, the time that the light transmittance begins to drop can be used to represent the time of the onset of liquid-liquid demixing. To carry out the light transmission experiment, a lamp was placed above the coagulation bath as light source and a light detector beneath the coagulation bath was used to measure the light transmittance.

\subsection{SEM}

The membrane structure was examined by a Hitachi (Model-S4800) scanning electron microscope (SEM). In SEM studies, the membrane sample was prepared by breaking the membrane in liquid nitrogen and was coated with $\mathrm{Au} / \mathrm{Pd}$ by sputtering.

\subsection{Pore size analysis}

For the measurement of the pore size distribution, a capillary flow porometer (CFP-1500AEXL, Porous Materials Inc.) was used. PVDF membrane sample was cut into $5 \mathrm{~cm}$ in diameter and wetted by the Galwick ${ }^{\mathbb{R}}$ solution (Porous Materials Inc.) first. The Galwick ${ }^{\mathbb{B}}$ is a standard wetting solution which surface tension is adjusted to $15.9 \mathrm{dyne} / \mathrm{cm}$. Then the fully wetted sample is placed in the sample chamber and the chamber is sealed. Gas (nitrogen gas) is then allowed to flow into the chamber behind the sample. When the pressure reached a point that could overcome the capillary action of the fluid within the largest pore, the bubble point was found. After determination of the bubble point, the pressure was increased and the flow rate was measured until all pores were empty and the sample was considered dry. Gas pressure and flow rates through the dry sample were also measured. The pore diameter is calculated according to the following equation:

$D=4 \gamma \cos \theta / P$

where $D$ is the pore diameter, $\gamma$ is the surface tension of the wetting solution, $\theta$ is the contact angle of wetting solution, and $P$ is differential gas pressure.

\subsection{Direct contact membrane distillation}

A schematic view of DCMD setup was shown in Fig. 1. A $3 \times 10^{4}$ ppm aqueous sodium chloride solution was used as feed solution in this work and the permeate side was distilled water. The selected porous membrane was tightly clamped between two acrylic plates, divided the system into the donor and receptor cell. The donor cell was below the porous membrane, the other cell above it. The aqueous sodium chloride solution was placed in donor cell (operation temperature was $30^{\circ} \mathrm{C}, 40^{\circ} \mathrm{C}$ and $50^{\circ} \mathrm{C}$ ) and the distilled water was in receptor cell (operation temperature 


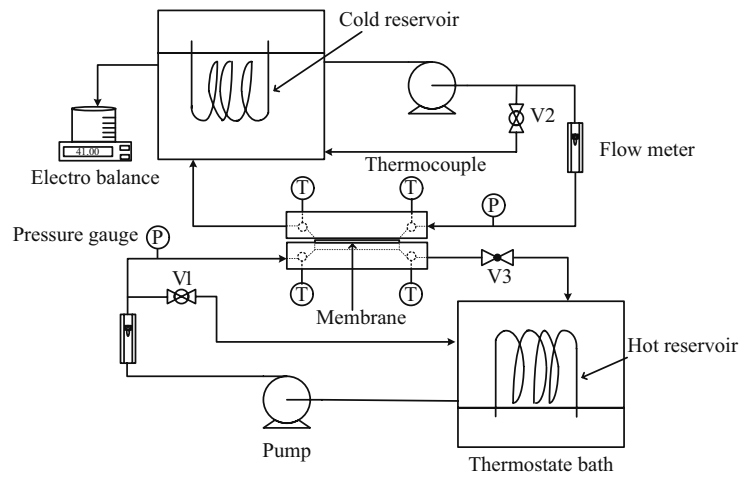

Fig.1. The schematic representation of direct contact membrane distillation setup.

was $20^{\circ} \mathrm{C}$ ). Both liquid were circulated in each cell by two independent pumps, respectively. The feed side velocity stood at $4 \mathrm{~L} / \mathrm{min}$, the permeate side was $7 \mathrm{~L} / \mathrm{min}$. The operation area of membrane was $1.02 \times 10^{-2} \mathrm{~m}^{2}$. Concentrations of the feed and permeate were determined with a SC-170 electric conductivity meter, purchased from SUNTEX Instrument limited company (Taiwan). The experimental obtained rejection, $R$, was defined as $R=\left[\left(C_{\mathrm{f}}-C_{\mathrm{p}}\right) / C_{\mathrm{f}}\right] \times 100 \%$, where $C_{\mathrm{f}}$ and $C_{\mathrm{p}}$ denote the sodium chloride concentration of feed solution and permeate, respectively.

\section{Results and discussion}

\subsection{Effect of coagulation condition on the membrane structure}

To prepare the PVDF membrane, the wet inversion phase separation process was carried out by using water and $n$-propanol as the nonsolvent, respectively. The surface and cross-section morphologies of these two PVDF membranes were shown in Fig. 2. The data in Fig. 2(a) depict that a dense surface can be formed as immersing the PVDF casting film into water bath. However, a distinct porous surface, with interconnected structure can be obtained by using $n$-propanol as precipitation bath as can be seen in Fig. 2(b). Moreover, a typical macrovoid structure can be formed in the cross-section of PVDF membrane which was coagulated by using water bath as shown in Fig. 2(c). A porous symmetric morphology of cross-section can be found in the $n$-propanol precipitated PVDF membrane as shown in Fig. 2(d). These phenomena might be related with the coagulation ability of the nonsolvent. The demixing rate of PVDF casting film in coagulation bath can be affected by the coagulation ability of coagulant. The solubility parameter difference between PVDF polymer and nonsolvent were $24.5\left(\mathrm{~J} / \mathrm{cm}^{3}\right)^{1 / 2}$ and $1.3\left(\mathrm{~J} / \mathrm{cm}^{3}\right)^{1 / 2}$ for water and $n$-propanol, respectively. Thus, the water presented more good nonsolvent then $n$-propanol for PVDF polymer solution.

Fig. 3 shows the light transmission curves of PVDF/NMP casting film which was immersed into water bath and $n$-propanol bath, respectively. The data in Fig. 3 reveal that by using the good nonsolvent (water) as the coagulant of PVDF/ NMP casting film, the phase separation process presented an instantaneous demixing. However, there was a delay demixing as using the poor nonsolvent (n-propanol) as the coagulant.

For the use of DCMD, porous membrane was necessary. The data in Fig. 2 reveal that a porous surface membrane can be prepared by using weak nonsolvent as the coagulant. The drawback of this membrane formation process was that this process might consume time during the precipitation period. Thus, we attempt to fabricate porous surface membrane by using dual-bath process in this section. Fig. 4 shows the morphology of PVDF membrane which was prepared by passing the PVDF casting film through $n$-propanol bath for $2 \mathrm{~s}$ and then was introduced into water bath.

The data in Fig. 4 reveal that the symmetric porous structure also can be formed as shown in Fig. 4. This morphology was similar to that formed by immersing in $n$-propanol bath for 1 day (Fig. 2). It meant that the porous PVDF membrane could be achieved by inserting a step with passing through alcohol only $2 \mathrm{~s}$ before 


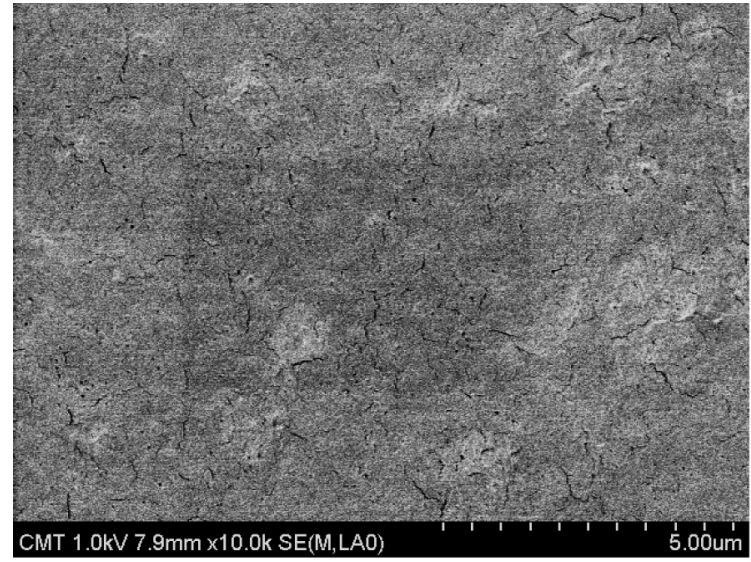

(a)

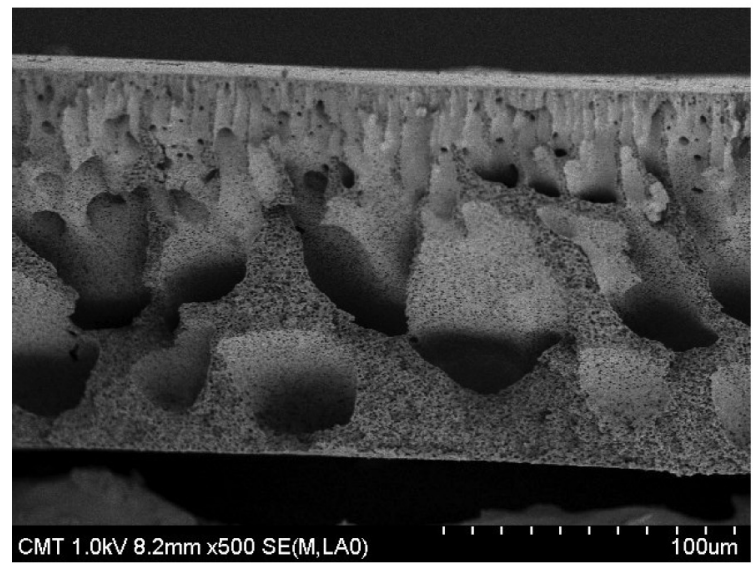

(c)

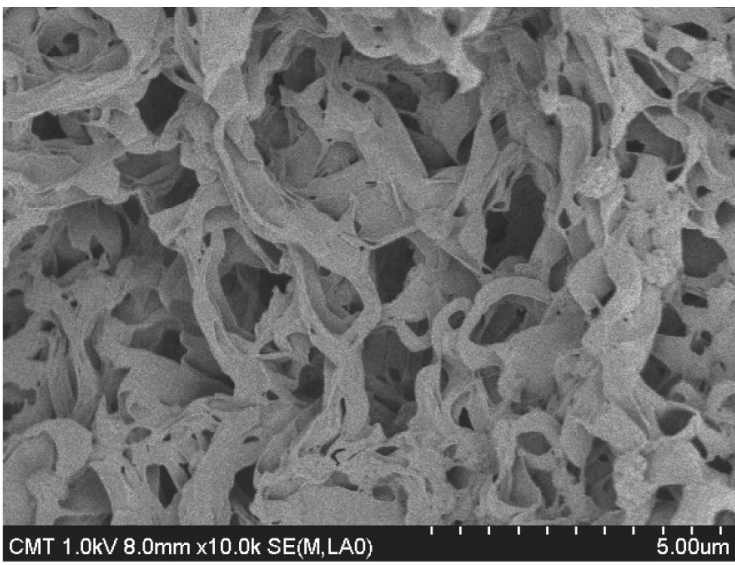

(b)

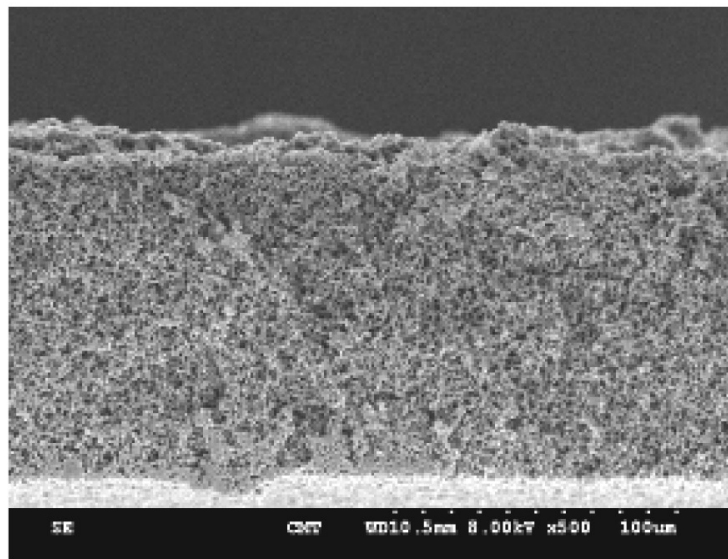

(d)

Fig. 2. SEM photographs of PVDF membranes prepared by using n-propanol and water as coagulant. (a) Water, surface; (b) $n$-propanol, surface; (c) Water, cross-section; (d) $n$-propanol, cross-section.

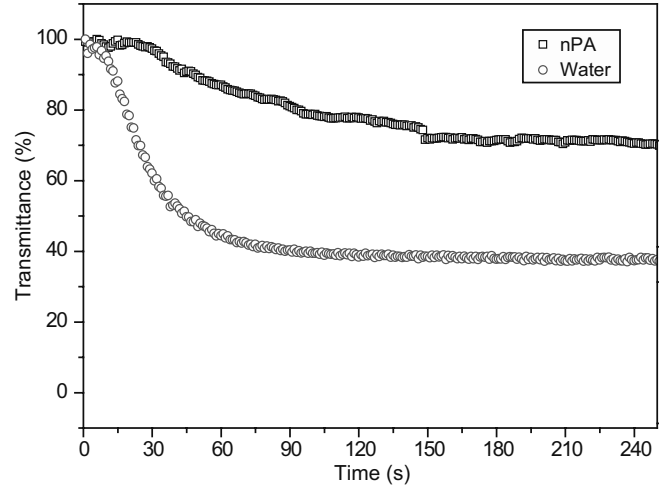

Fig. 3. The light transmission curves of PVDF casting

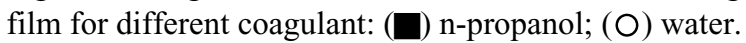

immersed into water bath. It was not necessary to prepare the porous structure by solidification in weak nonsolvent bath. This simple method can reduce the amount of alcohol and consumption time to fabricate the PVDF porous membrane.

\subsection{Effect of coagulation condition on the membrane hydrophobicity}

The hydrophobic property of PVDF membrane can be enhanced with roughness and porous surface. Table 1 summarized the water contact angles for the top surface of PVDF membranes 


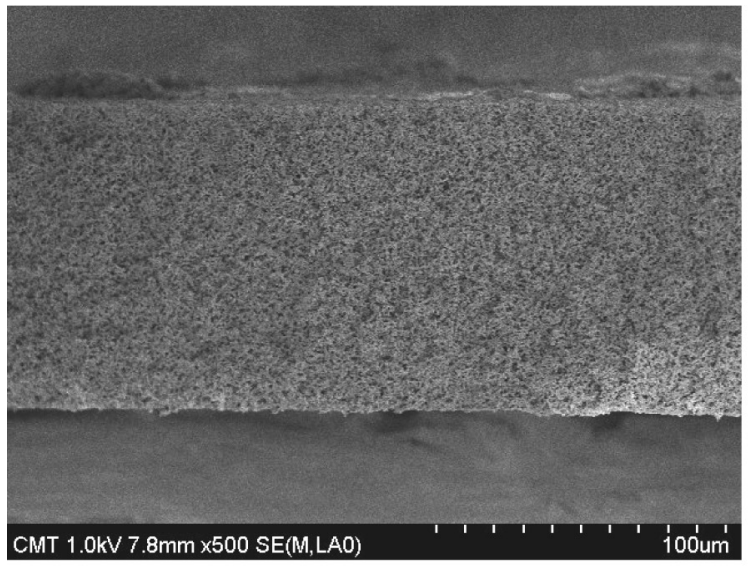

(a)

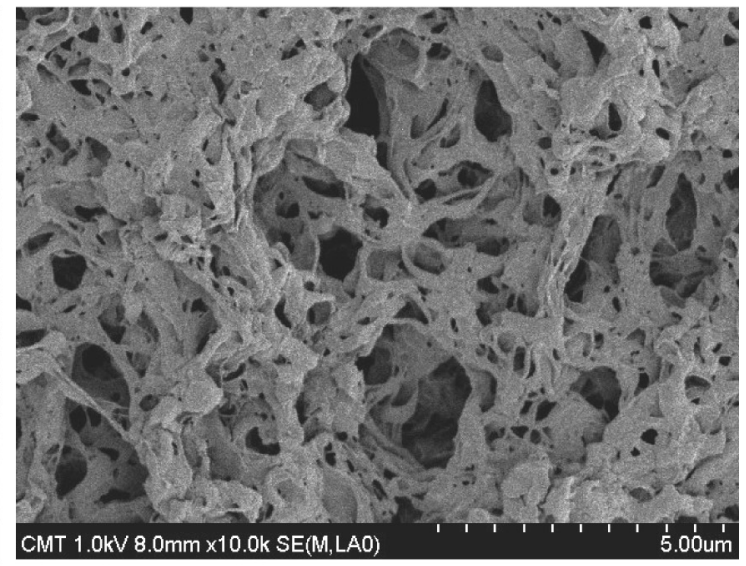

(b)

Fig. 4. SEM photographs of PVDF membranes prepared by immersing into n-propanol bath for $2 \mathrm{~s}$ and then immersed into water bath (dual coagulation method). (a) cross-section; (b) surface.

which were obtained by using the single coagulation bath (water bath or alcohol bath) or dual coagulation bath (alcohol bath for $2 \mathrm{~s}$ and then water bath). The water contact angle was about $84.0^{\circ} \pm 0.7^{\circ}$ for the dense surface of single water bath precipitated PVDF membrane as shown in Table 1. The water contact angle of the

Table 1

The water contact angle of PVDF membranes prepared by pass through various alcohol bath as a precursor for different time and then immersed into water bath. $\left(25^{\circ} \mathrm{C}\right)$

\begin{tabular}{llc}
\hline Coagulant & \multicolumn{2}{l}{ Contact angle value $\left(^{\circ}\right)$} \\
\cline { 2 - 3 } & $\begin{array}{l}\text { Dual-coagulation } \\
\text { bath }^{\text {a }}\end{array}$ & $\begin{array}{l}\text { Single-coagulation } \\
\text { bath }^{\mathrm{b}}\end{array}$ \\
\hline Water & & $84 \pm 1$ \\
Methanol & $139 \pm 1$ & $144 \pm 1$ \\
Ethanol & $138 \pm 1$ & $146 \pm 2$ \\
n-Propanol & $136 \pm 2$ & $148 \pm 2$ \\
n-Butanol & $137 \pm 1$ & $146 \pm 3$ \\
\hline
\end{tabular}

${ }^{a}$ The PVDF casting film was passed through alcohol for $2 \mathrm{~s}$ and then immersed into water bath.

${ }^{\mathrm{b}}$ The PVDF casting film was immersed into water or alcohol bath only. top surface of PVDF membranes obtained from immersed in single $n$-propanol bath was increased to $148 \pm 2^{\circ}$. It also can be found that the water contact angle was higher than $140^{\circ}$ by using alcohol as the single bath regardless of methanol, ethanol, $n$-propanol or $n$-butanol.

According to the SEM observation and water contact angle data, it can be concluded that porous surface morphology of PVDF membrane can be formed by using alcohol as nonsolvent and the hydrophobicity of PVDF membrane can be enhanced results from the porous structure. Even, the porous PVDF membrane obtained by passing through alcohol bath (methanol, ethanol, $n$-propanol or $n$-butanol) only $2 \mathrm{~s}$ and then immersed into water bath, the water contact angle value also increased higher than $135^{\circ}$.

\subsection{Performance test of the porous membrane}

Membrane pore size is an important factor to evaluate the membrane separation performance. The pore size distribution of the PVDF membrane prepared by passing through $n$-propanol bath for $2 \mathrm{~s}$ and then immersed into water precipitation bath was showed in Fig. 5. The data 


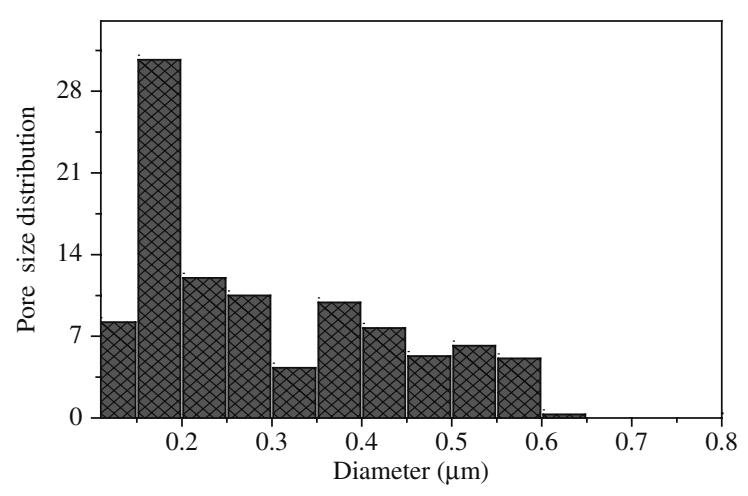

Fig. 5. The pore size distribution of PVDF membrane fabricated by passing through $n$-propanol bath for $2 \mathrm{~s}$ and then immersed in water bath.

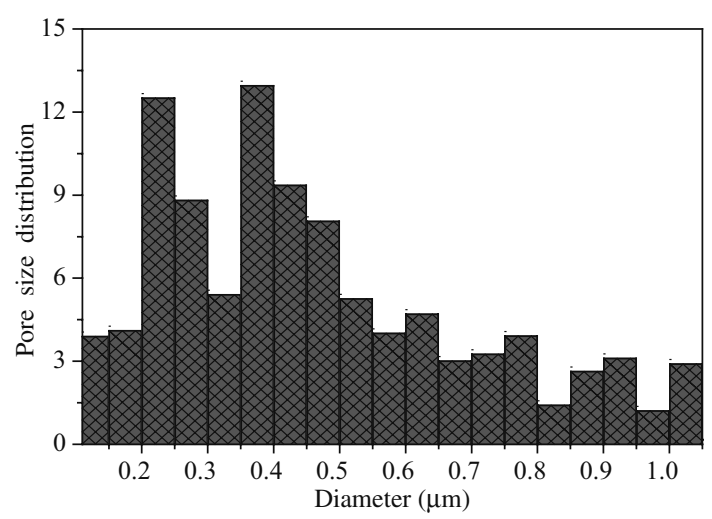

Fig. 6. The pore size distribution of PVDF membrane fabricated by immersed into $n$-propanol bath for 1 day. in Fig. 5 depict pore size was distributed in the range from 0.10 to $0.65 \mu \mathrm{m}$ and the centralized pore size of $0.15 \sim 0.3 \mu \mathrm{m}$ was over $50 \%$. Fig. 6 shows the pore size distribution of PVDF membrane obtained from immersed into $n$-propanol precipitation bath only. As can be seen in Fig. 5 and Fig. 6, the pore size distribution for $n$-propanol precipitated PVDF membrane was wider (from 0.1 to $1 \mu \mathrm{m}$ ) then dual-coagulation bath precipitated system. The centralized pore size of $0.2 \sim 0.5 \mu \mathrm{m}$ was over $50 \%$ for dual-coagulation bath precipitated system. The increasing of the immersed time in $n$-propanol bath can increased the pore size of PVDF membrane.

Membrane distillation is a separation process based on vapor of volatile components in an aqueous solution transported through the membrane pores, while the liquid cannot penetrate into the membrane pores due to its hydrophobicity. The feed solution in this work was a $3 \times 10^{4} \mathrm{ppm}$ aqueous sodium chloride solution and the permeate side was distilled water. Table 2 summarized the permeate flux and rejection coefficient of single $n$-propanol coagulated PVDF membrane, n-propanol/water dual-bath coagulated PVDF membrane and commercial PVDF membrane at 30 to $50^{\circ} \mathrm{C}$. The data in Table 2 shows the permeation flux increases with increasing operation temperature and the rejection coefficient was all near over $99.7 \%$. By comparing with the two PVDF membranes prepared in this study, the permeation flux of the dual-bath coagulated membrane was higher than the single bath one.

Table 2

The permeate flux and rejection coefficient of porous PVDF membrane

\begin{tabular}{lllllllll}
\hline Membrane & Thickness $(\mathrm{mm})$ & \multicolumn{2}{l}{ Permeate flux, $\left(\mathrm{kg} / \mathrm{m}^{2} \mathrm{~h}\right)$} & & \multicolumn{2}{l}{ Rejection coefficient, $R(\%)$} \\
\cline { 3 - 4 } & & $\left(30^{\circ} \mathrm{C}\right)$ & $\left(40^{\circ} \mathrm{C}\right)$ & $\left(50^{\circ} \mathrm{C}\right)$ & & $\left(30^{\circ} \mathrm{C}\right)$ & $\left(40^{\circ} \mathrm{C}\right)$ & $\left(50^{\circ} \mathrm{C}\right)$ \\
\hline Dual-bath & 0.11 & $1.27 \pm 0.04$ & $3.97 \pm 0.15$ & $9.29 \pm 0.23$ & & 99.90 & 99.93 & 99.98 \\
Single bath & 0.14 & $0.32 \pm 0.01$ & $2.95 \pm 0.19$ & $8.74 \pm 0.43$ & & 99.99 & 99.85 & 99.75 \\
PVDF $^{\mathrm{a}}$ & 0.20 & $0.74 \pm 0.01$ & $2.63 \pm 0.08$ & $5.37 \pm 0.24$ & & 99.97 & 99.95 & 99.96 \\
\hline
\end{tabular}

${ }^{\mathrm{a}}$ Immobilon-P $\mathrm{P}^{\mathrm{SQ}}$ (Millipore), pore size $0.2 \mu \mathrm{m}$. 
The permeation flux and rejection coefficient of the porous PVDF membranes fabricated by dual-bath coagulation method was close to commercial one.

\section{Conclusion}

In this paper, The porous PVDF membranes, with high hydrophobic surface (over than $140^{\circ}$ ) were prepared by using alcohol as nonsolvent (coagulant). The high hydrophobic property was facilely created by wet immersion method without modification with special low surface energy compounds and the need of special instruments. The porous PVDF membranes with the water contact angle of $135^{\circ}$ were also formed by passing through alcohol bath only $2 \mathrm{~s}$, such as methanol, ethanol, $n$-propanol or $n$-butanol and then immersed into water precipitation bath. The permeation flux and rejection coefficient of the porous PVDF membrane fabricated via dual-bath coagulated was close to commercial one.

\section{References}

[1] M. Mulder, Basic principles of membrane technology, 2nd edn., Kluwer Academic Publishers, London, 1996.

[2] K. Kurumada, T. Kitamura, N. Fukumoto, M. Oshima, M. Tanigaki and S. Kanazawa, Structure generation in PTFE porous membranes induced by the uniaxial and biaxial stretching operations, J. Membr. Sci., 149 (1998) 51-57.

[3] D.R. Lloyd, K.E. Kinzer and H.S. Tseng, Microporous membrane formation via thermally induced phase separation. I. Solid-liquid phase separation, J. Membr. Sci,. 52 (1990) 239-261.
[4] H. Matsuyama, T. Maki, M. Teramoto and K. Asano, Effect of polypropylene molecular weight on porous membrane formation by thermally induced phase separation, J. Membr. Sci., 204 (2002) 323-328.

[5] H. Matsuyama, T. Iwatani, Y. Kitamura, M. Tearamoto and N. Sugoh, Formation of porous poly(ethylene-co-vinyl alcohol) membrane via thermally induced phase separation, J. Appl. Polym. Sci., 79 (2001) 2449-2455.

[6] M.X. Shang, H. Matsuyama, T. Maki, M. Teramoto and D.R. Lloyd, Preparation and characterization of poly(ethylene-co-vinyl alcohol) membranes via thermally induced liquid-liquid phase separation, J. Appl. Polym. Sci., 87 (2003) 853-860.

[7] E. Fontananova, J.C. Jansen, A. Cristiano, E. Curcio and E. Drioli, Effect of additives in the casting solution on the formation of PVDF membranes, Desalination, 192 (2006) 190-197.

[8] D. Wang, K. Li and W.K. Teo, Porous PVDF asymmetric hollow fiber membranes prepared with the use of small molecular additives, J. Membr. Sci., 178 (2000) 13-23.

[9] D.M. Wang, T.T. Wu, F.C. Lin, J.Y. Hou and J.-Y. Lai, Novel method for controlling the surface morphology of polymeric membranes, J. Membr. Sci., 169 (2000) 39-51.

[10] D.J. Lin, C.L. Chang, T.C. Chen and L.P. Cheng, On the structure of porous poly(vinylidene fluoride) membrane prepared by phase inversion from water-NMP-PVDF system, J. Sci. Eng., 5 (2002) 95-98.

[11] D.J. Lin, H.H. Chang, T.C. Chen, Y.C. Lee and L.-P. Cheng, Formation of porous poly(vinylidene fluoride) membranes with symmetric or asymmetric morphology by immersion precipitation in the water/TEP/PVDF system, Eur. Polym. J., 42 (2006) 1581-1594.

[12] M. Peng, H. Li, L. Wu, Q. Zheng, Y. Chen and W. Gu, Porous poly(vinylidene fluoride) membrane with highly hydrophobic surface, J. Appl. Polym. Sci., 98 (2005) 1358-1363. 